\title{
BASES BIOLÓGICAS DEL DETERIORO DE LA FUNCIÓN COGNITIVA INDUCIDO POR LOS TRATAMIENTOS ANTINEOPLÁSICOS
}

\section{BIOLOGICAL BASES OF COGNITIVE IMPAIRMENT INDUCED BY ANTINOEPLASTIC TREATMENT}

\author{
Jaime Feliu', Sonia López-Santiago², Virginia Martínez-Marín', Ana Belén Custodio' y \\ Juan Antonio Cruzado² \\ I Servicio de Oncología Médica Hospital Universitario La Paz. IdIPAZ. RETICC RD06/0020/1022. \\ 2 Facultad de Psicología. Universidad Complutense de Madrid.
}

Resumen

A menudo los pacientes que reciben tratamiento con quimioterapia $y / 0$ radioterapia presentan problemas de memoria y de atención. Se desconocen los mecanismos exactos por los que esto se produce, aunque se han propuesto diversas explicaciones. Tanto la quimioterapia como la radioterapia poseen una acción tóxica directa en el SNC, especialmente sobre las células progenitoras neurales y las de la glía, que son las responsables de mantener la neurogénesis del hipocampo y la integridad de la sustancia blanca. A ello cabe añadir el daño que pueden inducir los tratamientos en la vascularización cerebral, la alteración de la respuesta inmune mediada por citoquinas, y los cambios hormonales ya sean secundarios a la quimioterapia o a la propia hormonoterapia. La intensidad de estos efectos estaría modulada por diversos factores genéticos que influyen sobre la capacidad de reparación neuronal, la farmacodinamia y la actividad de los neurotransmisores.

Palabras clave: Función cognitiva, cáncer, quimioterapia, radioterapia, chemobrain.

\section{Abstract}

Often patients treated with chemotherapy and/or radiotherapy suffer memory and attention problems. The exact mechanisms of action are unknown, although several explanations have been proposed. Both chemotherapy and radiotherapy have a direct toxic effect in the CNS, especially neural progenitor cells and the glia, which are responsible for maintaining neurogenesis in the hippocampus and white matter integrity. Besides the treatments can induce vascular damage in the brain, altering the immune response mediated by cytokines, and hormonal changes secondary to either chemotherapy or hormone therapy itself. The intensity of these effects would be modulated by several genetic factors that influence the ability of neuronal repair, pharmacodynamics and activity of neurotransmitters.

Keywords: Cognitive function, cancer, chemotherapy, radiotherapy, chemobrain.

\section{Corresponding author:}

J. Feliu, MD

Medical Oncology Service, Hospital Universitario La Paz.

$P^{\circ}$ de la Castellana 261

28046-Madrid

Spain

E-mail: jfeliu.hulp@salud.madrid.org 


\section{INTRODUCCIÓN Y MAGNITUD DEL PROBLEMA}

Los tratamientos antineoplásicos pueden causar, con frecuencia, efectos secundarios en el sistema nervioso central $(\mathrm{SNC})$. Esta neurotoxicidad incluye una amplia variedad de manifestaciones que pueden ser agudas o tardías. Entre ellas se encuentran las complicaciones vasculares, las crisis convulsivas, las alteraciones psicológicas y el deterioro cognitivo. Actualmente, se admite que hasta el 75\% de los pacientes con cáncer pueden experimentar una disminución de la función cognitiva (FC) durante o después del tratamiento antineoplásico ${ }^{(1)}$. Esta alteración a menudo es transitoria, pero puede ser persistente (meses o años) en el $17-35 \%$ de los casos. Su aparición se ha asociado tanto con la radioterapia (RT) craneal como con la quimioterapia (QT), ya sea sistémica o intratecal, o con la propia hormonoterapia $(\mathrm{HT})$. Se han acuñado términos como "chemobrain" o "chemofog" para referirse al deterioro de la memoria, capacidad de aprendizaje, concentración, razonamiento, atención, función ejecutiva y habilidades visuoespaciales que aparecen durante y después de finalizar la $\mathrm{QT}^{(2)}$. Afortunadamente, en la mayoría de las ocasiones el deterioro es sutil y mejora tras finalizar el tratamiento. Sin embargo, pequeños déficit pueden afectar a la calidad de vida del paciente y a su rendimiento, tanto en el trabajo como en el hogar. Además, en un subgrupo de pacientes este deterioro puede ser importante y persistente.

El procedimiento más utilizado para detectar el deterioro de la FC es la aplicación de diversos test neuropsicológicos. Sin embargo, no existe acuerdo sobre los dominios cognitivos que deben estudiarse y los test a utilizar ${ }^{(2)}$. Existe un cierto consenso en que debería evaluarse la atención, la velocidad de procesamiento, la memoria, la capacidad de aprendizaje, el lenguaje, la percepción visual, la función ejecutiva y las habilidades constructivas, motoras y de recuperación ${ }^{(3)}$. La Internacional Cognition and Cancer Task Force (ICCTF) recomienda utilizar, al menos, los siguientes test: Hopkins Verbal Learning Test-Revised (HVLT-R) para evaluar el lenguaje y el aprendizaje, el Trail Making Test (TMT) para estudiar la velocidad psicomotora y los aspectos de la función ejecutiva y el Controlled Oral Word Association (COWA) para medir la fluidez verbal, como reflejo de la función ejecutiva $^{(4)}$.

Las pruebas de neuroimagen (RNM y PET) son capaces de detectar los cambios morfológicos en el cerebro que se producen tras la administración de QT. Se ha descrito una disminución del volumen en estructuras cerebrales que están implicadas en la FC, como la corteza del lóbulo frontal, y una reducción tanto en la sustancia blanca como en la gris ${ }^{(2)}$. La realización de RNM funcional mientras se realizaba una tarea que requería memoria, mostró que los pacientes que habían recibido QT tenían una disminución de la activación de áreas del lóbulo frontal medio. Por el contrario, los pacientes que se quejaban de una disminución de sus funciones cognitivas presentaban un aumento de la activación de otras áreas del lóbulo frontal y del parietal. Mediante PET se ha podido demostrar que los pacientes tratados con QT y que realizaban tareas que requerían memoria, presentaban una disminución del metabolismo en la corteza cerebral, cerebelo y ganglios basales ${ }^{(5)}$. Igualmente, con técnicas de potenciales evocados, se pueden observar cambios en el electroencefalograma de pacientes tratados con dosis altas de QT. Concretamente se ha observado una reducción de la amplitud de P300 (se relaciona con la atención), y una actividad anormal de ondas alfa ${ }^{(6)}$. Estas alteraciones detectadas por electroencefalograma y técnicas de imagen sugieren que la propia QT tiene un impacto en la función cognitiva que es in- 
dependiente de la presencia del cáncer o de otros tratamientos.

El objetivo de esta revisión es describir los mecanismos que pueden estar implicados en los cambios de la FC asociados al tratamiento antineoplásico y los factores de riesgo que favorecen su aparición.

\section{QUIMIOTERAPIA Y DETERIORO COGNITIVO}

Se desconocen los mecanismos por los que se produce el deterioro cognitivo en pacientes con cáncer que reciben tratamientos con QT, aunque probablemente

Figura 1. Posibles mecanismos por los que los tratamientos antineoplásicos pueden alterar las funciones cognitivas

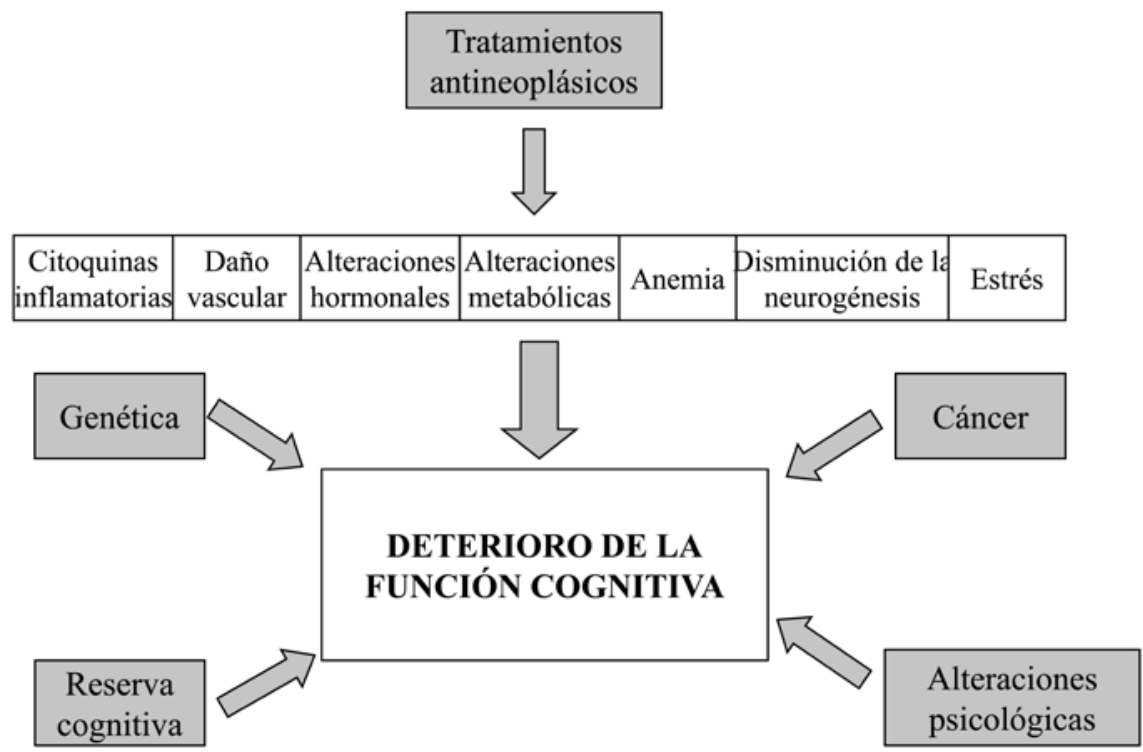

Tabla 1. Posibles mecanismos por los que los tratamientos antineoplásicos pueden alterar las funciones cognitivas

Citotoxicidad directa

Stem-cell neurales

Progenitores de la glía

Reducción de los niveles de factores neurotróficos

Acortamiento de la longitud de los telómeros

Disminución de neurotransmisores

Estrés oxidativo

Predisposición genética

Sistemas de reparación neuronales poco eficientes

Mecanismos reparadores de ADN poco eficaces

Bomba de eflujo de fármacos

Alteraciones en los niveles de citoquinas

Lesiones vasculares

Cambios hormonales 
su etiología sea múltiple (figura 1). Entre los diversos factores implicados podemos destacar la toxicidad neurológica de los citostáticos (daño directo sobre las neuronas y/o tejidos adyacentes, modificación de los niveles de neurotransmisores, fenómenos oxidativos), las lesiones vasculares cerebrales, la respuesta inmune inflamatoria (citoquinas), las alteraciones hormonales, la predisposición genética, etc. (tabla 1).

\section{Toxicidad neurológica inducida por los quimioterápicos}

Habitualmente se ha considerado que, salvo excepciones como el 5-fluorouracilo o el methotrexate, la mayoría de los citostáticos no atraviesan la barrera hematoencefálica. Sin embargo, sorprendentemente, casi todos estos fármacos pueden causar alguna toxicidad en el sistema nervioso central (SNC) (encefalopatía, toxicidad cerebelosa, ototoxicidad, leucoencefalopatía...). Con técnicas sofisticadas de medicina nuclear, se ha podido detectar la presencia de fármacos quimioterápicos radiomarcados en el SNC tras su administración sistémica (cisplatino, BCNU, paclitaxel...). Aunque sus concentraciones son demasiado bajas como para producir un efecto antineoplásico, no se puede descartar que alcancen el nivel necesario para deteriorar la $\mathrm{FC}^{(7)}$.

La mayoría de los fármacos quimioterápicos pueden causar daño celular y roturas de las cadenas de ADN, ya sea directamente (alquilantes, antimetabolitos) o bien indirectamente, a través de mecanismos oxidativos que incluyen, entre otros, la liberación de radicales libres (antraciclinas). Todo ello conduce a la apoptosis celular. Tradicionalmente se considera que estos fármacos actúan preferentemente sobre las células que se dividen rápidamente, como es el caso de las células endoteliales y las de la glía, pero no sobre las neuronas. Estudios más recientes han de- mostrado que en los cerebros adultos de los mamíferos existen células madre neurales capaces de auto-renovarse y que dan lugar a neuronas, células de la astroglía y de la oligodendroglía. Todas estas células precursoras se denominan células neurales progenitoras. Una de las zonas donde se encuentran estas células progenitoras es el hipocampo. Actualmente se considera que el proceso de neurogénesis en la zona del hipocampo es fundamental para la correcta realización de algunas de las funciones de memoria y aprendizaje. Este proceso puede afectarse negativamente por la quimioterapia, la radioterapia, ciertos estados inflamatorios y por la liberación de glucocorticoides en situaciones de estrés ${ }^{(8)}$.

Se ha podido demostrar que las células más sensibles a la acción de la QT son las células progenitoras neurales y los oligodendrocitos maduros (células formadoras de mielina). Por el contrario, los astrocitos maduros y las neuronas son menos vulnerables.

En diversos modelos animales se ha detectado una relación consistente entre la administración de quimioterapia y la alteración de algunos dominios $\operatorname{cognitivos}^{(9)}$. Así por ejemplo, El 5FU y el MTX causan en ratones déficits agudos en ciertas funciones cognitivas que requieren la integridad del hipocampo y del lóbulo frontal ${ }^{(3)}$. Más recientemente se ha señalado que el 5FU puede causar toxicidad en los oligodendrocitos y en las células progenitoras neurales. La supresión continuada de la proliferación de las células progenitoras se asoció con un aumento de la muerte celular. Además, también se detectó que podía inducir en el cerebro del ratón una inflamación aguda y daño vascular, lo que puede favorecer el desarrollo tardío de lesiones desmielinizantes, detectables a los 6 meses. Igualmente, la administración de cisplatino, carmustina y arabinósido de citosina puede producir un aumento de la muerte celular y una disminución de la división celular también 
en la zona subventricular, el girus dentado del hipocampo y el cuerpo calloso. Estos cambios se pudieron detectar con dosis inferiores a las necesarias para producir efectos antineoplásicos.

Por otra parte, se ha observado que las células proliferativas del girus dentado pueden evolucionar a neuronas involucradas en la memoria y el aprendizaje. Para ello se requiere la presencia de factores neurotróficos como el factor neurotrópico derivado del cerebro (BDNF). Recientemente se ha comunicado que la administración de 5FU puede reducir los niveles de BDNF y de neurotropina en el hipocampo, e interferir con la neurogénesis ${ }^{(11)}$. Además, los quimioterápicos pueden modificar las funciones celulares de las células progenitoras neurales ${ }^{(10,12)}$.

Otro aspecto a considerar es que muchos citostáticos también alteran la sustancia blanca. Así por ejemplo, el MTX puede dañar las células progenitoras de la glía que se diferencian en oligodendrocitos $y$ astrocitos, y que resultan críticos para la formación de la mielina y la integridad de la sustancia blanca.

En este contexto adquieren una especial importancia los mecanismos de reparación del ADN. Se ha descrito que la disminución de la capacidad de reparar el daño del ADN mitocondrial se relaciona con un aumento de la apoptosis en los cultivos de células neurales ${ }^{(13)}$. Alternativamente, también implicaría un bloqueo de la transcripción génica, lo que supone la pérdida de ciertas funciones celulares. Cabe especular con la posibilidad de que las alteraciones en los mecanismos reparadores del ADN puedan predisponer tanto a padecer un cáncer como a presentar cuadros neurodegenerativos ${ }^{(7)}$.

\section{Daño vascular}

Tanto la QT como la RT pueden lesionar los vasos sanguíneos, lo que re- duce el flujo en los pequeños vasos del cerebro, ya sea por sus efectos directos, por el estrés oxidativo o por la formación de pequeños $\operatorname{coágulos}^{(7)}$. Además, se ha descrito que la administración de MTX puede disminuir la densidad vascular en el hipocampo ${ }^{(14)}$.

\section{Citoquinas}

Las citoquinas pro-inflamatorias IL-1, IL-6 y TNFalfa atraviesan la barrera hematoencefálica y pueden causar astenia, anorexia, disminución de la función cognitiva, etc. ${ }^{(15)}$. Actualmente se considera que las citoquinas no sólo regulan la inflamación, sino que también pueden desempeñar un papel importante a nivel del SNC. Se ha escrito que intervienen en la modulación de la función de las neuronas y de las células de la glía, en la reparación neural y en el metabolismo de la dopamina y la serotoni$\mathrm{na}^{(7)}$. De hecho, en algunas enfermedades neurodegenerativas como la enfermedad de Parkinson, la enfermedad de Alzheimer, etc., se ha visto una desregulación de las citoquinas. Igualmente, la administración de tratamientos con IL-2 o interferón se ha asociado a depresión, astenia y deterioro cognitivo. Este último se manifestaba como disminución de la velocidad de procesamiento, función ejecutiva, habilidad espacial y del tiempo de reacción ${ }^{(16,17)}$. Se ha propuesto una mutua influencia entre el cerebro y las citoquinas pro-inflamatorias periféricas y centrales (sistema inmune). Las citoquinas informan al cerebro de los daños y lesiones que sufre el organismo, comunicándose con él por diversos mecanismos. Al recibir esta información se desencadena una "respuesta de enfermedad" tanto a nivel psíquico (ej., variación en el patrón de sueño) como conductual (ej., reducción de actividad), que facilita el ahorro energético y permite al sistema inmune luchar contra los agentes infecciosos o dañinos para el organismo. Además, 
las citoquinas pueden influir en el humor, el sistema sensorial y la consolidación de la memoria. Así por ejemplo, en pacientes con tumores hematológicos se ha detectado una asociación entre los niveles elevados de IL-6 y una peor función ejecutiva. Por el contrario, las concentraciones altas de IL-8 se relacionaban con un mejor funcionamiento de la memoria ${ }^{(18)}$. Mientras en las pacientes diagnosticadas de cáncer de mama se observó una cierta relación entre los niveles de citoquinas y la función cognitiva ${ }^{(19)}$, en los enfermos con cáncer de colon esto no se pudo demostrar ${ }^{(20)}$.

Cabe destacar un estudio hecho en ratones expuestos a adriamicina en el que se observó que este fármaco producía un aumento de los niveles de TNFalfa en la corteza cerebral y el hipocampo, con una hiperactivación de la microglía, una inducción del estrés oxidativo, disfunción mitocondrial y un aumento de la muerte celular. Todo ello, a pesar de que no se detectó adriamicina en esos cerebros ${ }^{(21)}$. Por otra parte, recientemente se ha comunicado una relación entre el TNFr2 circulante y el deterioro de la función cognitiva en pacientes con cáncer de mama que habían recibido QT. Además, se observó una relación entre la elevación del TNFr2 en sangre y una disminución del metabolismo en la zona inferior izquierda del girus frontal, que incluía el área de Bro$\mathrm{Ca}^{(22)}$.

Así pues, existen evidencias que sugieren que la QT puede aumentar los niveles de ciertas citoquinas ${ }^{(22,23)}$ y que esta respuesta no es uniforme, sino que se elevan de forma diferente en función del tipo de QT empleada ${ }^{(24)}$. Por todo ello, no se puede descartar que puedan jugar un papel importante en el deterioro cognitivo relacionado con la QT. De hecho, se ha sugerido que aunque la QT causa neurotoxicidad, la inflamación podría actuar de mediador reduciendo la transmisión neural ${ }^{(1)}$.

\section{Predisposición genética}

Los factores genéticos pueden desempeñar un papel fundamental en el desarrollo del deterioro de la FC relacionado con el tratamiento. Desde hace algunos años se conoce la relación entre los polimorfismos de los genes implicados en los procesos reparadores y la vulnerabilidad para desarrollar esta complicación. Quizás uno de los más estudiados son los polimorfismos de la apolipoproteína E (APOE). La APOE es una glicoproteina compleja que interviene en la captación, transporte y distribución de lípidos. Parece que juega un papel importante en la reparación neuronal. Posee tres alelos (E2, E3 y E4). En el ser humano, el alelo E4 se ha relacionado con una amplia variedad de trastornos que cursan con disfunción cognitiva y que van desde personas normales que refieren pérdida de memoria hasta la enfermedad de Alzheimer, pasando por evoluciones desfavorables tras un ACVA o un trauma cerebral ${ }^{(25)}$. En un estudio se observó que los pacientes que habían recibido QT y que tenían una expresión polimórfica del alelo E4 del gen APOE presentaban una disminución significativa de la memoria visual y espacial, así como una tendencia hacia una menor función ejecutiva en comparación con los que no eran portadores de este alelo ${ }^{(26)}$. Se desconoce el mecanismo exacto por el que esto ocurre, aunque se cree que el alelo E4 del APOE sería menos eficaz que el alelo E3 para promover la reparación neuronal ${ }^{(7)}$. También se ha observado que el volumen del hipocampo de los portadores del alelo E4 es más pequeño que el de la población normal, lo cual parece importante para el adecuado funcionamiento de la memoria ${ }^{(27)}$. Otro tanto puede ocurrir con los genes reparadores de ADN. Concretamente, las personas con mecanismos reparadores de ADN deficientes tendrán más dificultad para recuperarse de la agresión que supone para el ADN 
los tratamientos con QT o RT. Igualmente pueden tener interés los polimorfismos del BDNF. La sustitución del aminoácido valina por metionina se ha relacionado con un peor resultado en los test de memoria y función ejecutiva $y$, además, con un hipocampo más pequeño ${ }^{(28)}$. No obstante, se desconoce si este polimorfismo puede favorecer o no la disminución de la FC en relación con el tratamiento antineoplásico.

Por otra parte, ciertos polimorfismos pueden alterar la farmacodinámica de los quimioterápicos y predisponer a una mayor toxicidad, ya sea mediante el aumento de la permeabilidad de la barrera hematoencefálica o bien favoreciendo una mayor exposición a los fármacos debido a unos mecanismos detoxificadores menos eficientes. Así por ejemplo, el gen de resistencia a múltiples drogas (MDR1) codifica la glicoproteína -P (P-gp) que desempeña un papel clave para transportar productos tóxicos al exterior de la célula. Por ello, sus niveles pueden influir en la exposición del cerebro a la QT.

Igualmente parecen importantes los polimorfismos relacionados con los neurotransmisores. Entre ellos, uno de los más estudiados es el polimorfismo de la catecol-O-metiltransferasa (COMT). Esta enzima interviene en la degradación de las catecolaminas mediante la metilación de la dopamina, la adrenalina y la noradrenalina, siendo la responsable del $60 \%$ de la degradación metabólica de la dopamina. Se ha comunicado que los pacientes tratados con quimioterapia que eran portadores del alelo valina de la COMT obtenían una puntuación peor en los test de memoria espacial y visual que los que eran homocigotos para el alelo metionina ${ }^{(7)}$.

\section{Cambios hormonales}

Tanto los estrógenos como los andrógenos poseen efectos antioxidantes y neuroprotectores, promueven la supervivencia de las células del cerebro y aumentan el flujo sanguíneo cerebral y el transporte de glucosa al cerebro ${ }^{(29)}$. Además, modulan la plasticidad estructural del hipocampo ${ }^{(30)}$ y ejercen un efecto beneficioso sobre los neurotransmisores que están implicados en el proceso cognitivo. Cabe señalar que en el cerebro se pueden localizar receptores de estrógenos y andrógenos en diversas zonas involucradas en la memoria y el aprendizaje, como son el tálamo, el hipocampo y la corteza cerebral ${ }^{(31)}$. De hecho, un aspecto que puede tener importancia en la función cognitiva son los polimorfismos de los genes de estos receptores. Así por ejemplo, algunos estudios han relacionado los polimorfismos del gen del receptor alfa de estrógenos (ESR1) con el riesgo de desarrollar la enfermedad de Alzheimer $^{(32)}$. Igualmente, se han descrito polimorfismos en el gen del receptor de andrógenos que parecen relacionarse con la $\mathrm{FC}^{(33)}$. Por todo ello, es interesante investigar la influencia de estos polimorfismos en el deterioro de la FC asociado a los tratamientos antineoplásicos.

A pesar de que estas observaciones parece lógico esperar que el descenso de los niveles hormonales inducidos por la QT o la HT afecte negativamente la $\mathrm{FC}^{(2)}$, los resultados de los estudios clínicos resultan algo más confusos. Mientras que en algunos trabajos se ha sugerido que el tamoxifeno sólo o asociado a QT puede contribuir al deterioro de la $\mathrm{FC}^{(34,35)}$, las evidencias resultan más débiles para los inhibidores de la aromatasa ${ }^{(36,37)}$ y el bloqueo androgénico ${ }^{(31,38)}$.

\section{Anemia}

Se trata de una complicación frecuente entre los pacientes con cáncer, ya sea producida por el propio tumor o por su tratamiento. Suele cursar con astenia y una disminución de la oxigenación cerebral, lo que puede favorecer el deterioro de la 
$\mathrm{FC}^{(39)}$. Sin embargo, resulta poco probable que tenga alguna responsabilidad en el mantenimiento de esta complicación tras la finalización del tratamiento, ya que suelen normalizarse sus cifras. Nuestro grupo, en un estudio previo, no pudo detectar ninguna relación entre la cifra de hemoglobina y la función cognitiva ${ }^{(40)}$.

\section{RADIOTERAPIA Y DETERIORO COGNITIVO}

Se han comunicado problemas cognitivos en el 22-30\% de los pacientes tratados con RT, y suelen desarrollarse 2-5 años después de haber finalizado el tratamiento ${ }^{(41)}$. Cabe señalar que la aparición de esta complicación depende de la edad (más frecuente por debajo de los 6 años y por encima de los 60 años), de la dosis y técnica empleada, del volumen irradiado, de la situación de la enfermedad y de la existencia de factores de riesgo vascular ${ }^{(42,43)}$. Así por ejemplo, cuando se emplea RT craneal profiláctica, se estima que un $20 \%$ de los pacientes sufre un deterioro transitorio de la FC que afecta principalmente a la función ejecutiva y al lenguaje ${ }^{(44,45)}$. Un estudio reciente ha comunicado que al año de finalizar la RT es posible detectar una disminución significativa de la memoria ${ }^{(46)}$. Cuando se irradian metástasis cerebrales, este deterioro puede detectarse hasta en el $50 \%$ de los pacientes y tiene un carácter más permanente ${ }^{(44)}$, mientras que si se trata de tumores cerebrales, puede encontrarse hasta en el $90 \%$ de los $\operatorname{casos}^{(47)}$. Por otra parte, también tiene importancia la técnica utilizada. En un ensayo que comparó la administración de radiocirugía frente a radiocirugía más RT holocraneal, se observó que el deterioro de la capacidad de aprendizaje y memoria a los 4 meses de finalizar el tratamiento era del $20 \%$ vs $64 \%{ }^{(48)}$.

$\mathrm{Al}$ igual que con la QT, se han sugerido diversos factores que pueden explicar el desarrollo de esa complicación, aunque su mecanismo exacto tampoco se conoce. Por una parte, la propia presencia del tumor o de sus metástasis puede justificar este problema. El fenómeno denominado diaschisis, hace referencia a la pérdida de función de un área del cerebro que está conectada con otra zona que se encuentra dañada. La diaschisis se ha descrito en diversas patologías, entre las que figuran los tumores cerebrales. Además, la neoplasia también puede alterar el flujo sanguíneo y el metabolismo de la zona, lo que puede contribuir al desarrollo de esta complicación $^{(47)}$. Por otra parte, la RT produce daños en el tejido cerebral que pueden ser o no reversibles. Tradicionalmente se distinguen tres fases en la respuesta cerebral a la RT holocraneal, dependiendo del tiempo de comienzo de los síntomas. Los efectos agudos aparecen durante la primera semana de la RT, se manifiestan como déficit neurológicos focales y son reversibles. Se atribuyen al edema cerebral, ya que suelen ceder con corticoides. Los efectos subagudos se desarrollan de 1-6 meses tras finalizar la RT. Se caracterizan por somnolencia, astenia y disfunción cognitiva. Esta última cursa con una disminución de la velocidad de procesamiento, de la memoria verbal, de la función ejecutiva y de la atención, todo ello, en relación con una disfunción del lóbulo frontal ${ }^{(43)}$. Se atribuye a una desmielinización difusa. Los efectos tardíos son los que se manifiestan después de 6 meses de haber finalizado la RT. Se expresan como un déficit progresivo de memoria, atención, procesamiento visuo-motor, etc. El mecanismo subyacente parece ser una disfunción del hipocampo ${ }^{(43)}$. En casos severos, puede detectarse una alteración de la sustancia blanca, debido al daño que se produce en los pequeños vasos. Esta complicación es progresiva, irreversible y cursa con desmielinización y necrosis ${ }^{(49)}$.

Recientemente se ha observado que la RT, al igual que la QT, también puede dañar a las células madre y a las neuronas 
progenitoras neurales del hipocampo. En modelos animales se ha demostrado que la RT aumenta la apoptosis y disminuye la proliferación y la diferenciación celular en la región neurogénica del hipocampo. Aunque estos cambios son más evidentes a largo plazo, a las pocas horas de administrar RT a dosis relativamente bajas, ya es posible observar una significativa pérdida de las células del hipocampo. Además, las células precursoras que sobreviven presentan una menor capacidad para diferenciarse a neuronas maduras ${ }^{(50)}$. En contraste con los efectos de la RT sobre la neurogénesis, la gliogénesis resiste mejor la acción de la RT. Por otra parte, los resultados de algunos experimentos sugieren que el microambiente desempeña un papel clave en la inhibición de la neurogénesis. De hecho, las células progenitoras neurales obtenidas de un hipocampo irradiado mantienen su capacidad para diferenciarse. Sin embargo, las células progenitoras no irradiadas cuando se implantan en un hipocampo irradiado no son capaces de diferenciarse $^{(51)}$. Esto se cree que es debido a que se altera la estrecha relación anatómica existente en la región neurogénica entre las células progenitoras y la microvascularización. A ello también contribuiría la fuerte inflamación que provoca la RT sobre la microglía. La RT activaría la microglía local y la producción de citoquinas proinflamatorias, como la IL-6, que contribuirían al bloqueo de la diferenciación neuronal(52).

Recientemente, estas observaciones experimentales se han podido confirmar en la clínica. En un estudio que realizó un análisis comparativo entre el tejido cerebral obtenido de pacientes diagnosticados de meduloblastoma que habían sido tratados mediante cirugía, RT y QT, y el procedente de un grupo control de la misma edad y sexo, se observó la casi completa eliminación de la neurogénesis del hipocampo en los primeros ${ }^{(53)}$.
Así pues, cabe afirmar que muchos de los efectos tardíos de la RT cerebral serían el resultado de los daños causados sobre las células madre y las células progenitoras neuronales, a lo que se añadiría la respuesta inflamatoria y las alteraciones del metabolismo ${ }^{(43)}$.

\section{CONCLUSIONES Y PERSPECTIVAS DE FUTURO}

Actualmente se considera que la base celular del deterioro cognitivo relacionado con el tratamiento antineoplásico, ya sea RT o QT, reside en la alteración de la neurogénesis y gliogénesis postnatal del SNC. Esto daría lugar a cambios en el hipocampo y en la sustancia blanca subcortical, que se traducirían en un deterioro de la memoria episódica (verbal y visual) y un cuadro parecido a la demencia subcortical (procesamiento lento de la información, disminución de la atención, etc. ${ }^{(11)}$. El hecho de que no todos los pacientes que reciben QT y/o RT desarrollan esta complicación indica que además de los efectos directos del tratamiento sobre el SNC existen otros factores individuales que modulan su aparición. Entre ellos cabe destacar los factores genéticos, los cambios hormonales, la reserva cognitiva del enfermo, la edad, aspectos psicológicos, etc. Por ello, probablemente sea necesaria la concurrencia de uno o varios de estos factores para que se produzca un deterioro duradero de la FC.

A pesar de los esfuerzos que se están realizando en este campo, todavía queda mucho por investigar. Es necesario profundizar en el conocimiento de los mecanismos implicados en el desarrollo de esta toxicidad. Este aspecto resulta crítico para poder diseñar fármacos neuroprotectores selectivos que eviten el daño que pueden causar los tratamientos antineoplásicos en las neuronas sanas. Otra posible aproximación terapéutica consistiría en la estimulación de la neurogénesis, de la gliogénesis 
y de los mecanismos reparadores endógenos. Esto permitiría evitar el desarrollo de la toxicidad tardía, como puede ser la relacionada con la desmielinización.

\section{REFERENCIAS BIBLIOGRÁFICAS}

1. Janelsins MC, Kohli S, Mohile SG, Usuki K, Ahles TA, Morrow GR. An update on cancer- and chemotherapy- related cognitive dysfunction: current status. Sem Oncol 2011; 38:431-8. Doi:10.1053/j.seminoncol.2011.03.014

2. Argyriou AA, Assimakopoulos K, Iconomou G, Giannakopoulou F, Kalofonos HP. Either called "chemobrain" or "chemofog", the long-term chemotherapy-induced cognitive decline in cancer survicors is real. J Pain Symptom Manag 2011 (in press). Doi:10.1016/j.jpainsymman.2010.04.021

3. Vardy J, Wefel JS, Ahles T, Tannock IF, Schagen SB. Cancer and cancer-therapy related cognitive dysfunction: an international perspective from the Venice cognitive workshop. Ann Oncol 2008; 19:623-9. Doi:10.1093/annonc/mdm500

4. Wefel JS, Vardy J, Alhes TA, Schagen SB. International Cognition and Cancer Task Force recommendations to harmonise studies of cognitive function in patients with cancer. Lancet Oncol 2011; 12:703-8. Doi:10.1016/S1470-2045(10)70294-1

5. Silverman DH, Dy CJ, Castellon SA, Lai J, Pio BS, Abraham L, et al. Altered frontocortical, cerebellar, and basal ganglia activity in adjuvant-treated breast cancer survivors 5-10 years after chemotherapy. Breast Cancer Res Treat 2007; 103:303-11. Doi:10.1007/s10549-006-9380-z

6. Kreukels BP, Schagen SB, Ridderinkhof KR, Boogerd W, Hamburger HL, Muller MJ et al. Effects of highdose and conventionaldose adjuvant chemotherapy on long-term cognitive sequelae in patients with breast cancer: an electrophysiologic study. Clin Breast Cancer 2006; 7:67-78. Doi:10.3816/ CBC.2006.n.015
7. Ahles TA, Saykin AJ. Candidate mechanisms for chemotherapy induced cognitive changes. Nat Rev Cancer 2007; 7:192-201. Doi:10.1038/nrc2073

8. Monje M, Dietrich J. Cognitive side effects of cancer therapy demonstrate a functional role for adult neurogenesis. Behav Brain Res 2011 (en prensa). Doi:10.1016/j. bbr.2011.05.012

9. Seigers R, Fardell JE. Neurobiological basis of chemotherapy-induced cognitive impairment: a rebiew of rodent research. Neurosci Biobehav Rev 2011; 35:729-41. Doi:10.1016/j.neubiorev.2010.09.006

10. Dietrich J, Han R, Yang Y, Mayer-Pröschel M, Noble M. CNS progenitor cells and oligodendrocytes are targets of chemotherapeutic agents in vitro and in vivo. J Biol 2006;5:22. Doi:10.1186/jbiol50

11. Mustafa S, Walker A, Bennett G, Wigmore PM. 5-Fluorouracil chemotherapy affects spatial working memory and newborn neurons in the adult rat hippocampus. Eur J Neurosci 2008;28:323-30. Doi:10.1111/ j.1460-9568.2008.06325.x

12. Hyrien O, Dietrich J, Noble M. Mathematical and experimental approaches to identify and predict the effects of chemotherapy on neuroglial precursors. Cancer Res 2010;70:10051-9. Doi:10.1158/00085472.CAN-10-1400

13. Harrison JF, Hollensworth SB, Spitz DR, Copeland WC, Wilson GL, LeDoux SP. Oxidative stress induced apoptosis in neurons correlates with mitochondrial DNA base excision repair pathway imbalance. Nucleic Acid Res 2005;33:4660-71. Doi:10.1093/nar/gki759

14. Seigers R, Timmermans J, van der Horn HJ, de Vries EF, Dierckx RA, Visser L, et al. Methotrexate reduces hippocampal blood vessel density and activates microglia in rats but does not elevate central cytokine release. Behav Brain Res 2010;207:265-72. Doi:10.1016/j.bbr.2009.10.009

15. Seruga B, Zhang H, Bernstein LJ, Tannock IF. Cytokines and their relationship onto 
the symptoms and outcome of cancer. Nat Rev Cancer 2008;8:887-99. Doi:10.1038/ nrc2507

16. Scheibel RS, ValentineAD, O'Brien S, Meyers CA. Cognitive dysfunction and depression during treatment with interferon-alpha and chemotherapy. J Neuropsychiatry Clin Neurosci 2004; 16: 185-91. Doi:10.1176/ appi.neuropsych.16.2.185

17. Capuron L, Ravaud A, Dantzer R. Timing and specificity of the cognitive changes induced by interleukin-2 and interferonalpha treatments in cancer patients. Psychosom Med 2001; 63:376-86.

18. Meyers CA, Albitar M, Estey E. Cognitive impairment, fatigue, and cytokine levels in patients with acute myelogenous leukemia or myelodysplastic syndrome. Cancer 2005;104:788-93. Doi:10.1002/cncr.21234

19. Booth CM, Vardy J, Crawley A, Rourke S, Pond G, Wagner L, et al. Cognitive impairment associated with chemotherapy for breast cancer: an exploratory case-control study. Proc ASCO 2006;24:8501.

20. Vardy J, Booth C, Pond GR, Galica J, Park A, Dhillon $\mathrm{H}$, et al. Cytokine levels in patients with colorectal cancer and breast cancer and their relationship to fatigue and cognitive function. Proc ASCO 2007;25:9070.

21. Tangpong J, Cole MP, Sultana R, Joshi G, Estus S, Vore $M$, et al. Adriamcin-induced TNF-alpha-mediated central nervous system toxicity. Neurobiol Dis 2006;23:12739. Doi:10.1016/j.nbd.2006.02.013

22. Ganz PA, Castellon SA, Silverman DH, Kwan L, Bower JE, Irwin MR, et al. Does circulating tumor necrosis factor (TNF) play a role in post-chemotherapy cerebral dysfunction in breast cancer survivors (BCS)?. Proc Am Soc Clin Oncol 2011; Abstract No: 9008

23. Tsavaris N, Kosmas C, Vadiaka M, Kanelopoulos P, Boulamatsis D. Immune changes in patients with advanced breast cancer undergoing chemotherapy with taxanes. Br J Cancer 2002;87:21-7. Doi:10.1038/ sj.bjc. 6600347
24. Janelsins MC, Mustian KM, Palesh OG, Mohile SG, Peppone LJ, Sprod LK, et al. Differential expression of cytokines in breast cancer patients receiving different chemotherapies: implications for cognitive impairment research. Support Care Cancer 2011. En prensa. Doi: 10.1007/s00520011-1158-0

25. Nathoo N, Chetty R, van Dellen JR, Barnett $\mathrm{GH}$. Genetic vulnerability following traumatic brain injury: The role of apolipoprotein E. Mol Pathol 2003;56:132-6. Doi:10.1136/mp.56.3.132

26. Ahles TA, Saykin AJ, Noll WW, Furstenberg CT, Guerin S, Cole B et al. The relationship of APOE genotype to neuropsychological performance in long-term cancer survivors treated with standard dose chemotherapy. Psychoncology 2003;12:612-9. Doi:10.1002/pon.742

27. Lind J, Larsson A, Persson J, Ingvar M, Nilsson LG, Bäckman L, et al. Reduced hippocampal volume in non-demented carriers of the apolipoprotein E epsilon4: relation to chronological age and recognition memory. Neurosci Lett 2006;396:23-7. Doi:10.1016/j.neulet.2005.11.070

28. Richter-Schmidinger $T$, Alexopoulos $P$, Horn M, Maus S, Reichel M, Rhein C, et al. Influence of brain-derived neurotrophicfactor and apolipoprotein E genetic variants on hippocampal volume and memory performance in healthy young adults. J Neural Transm 2011;118:249-57. Doi:10.1007/ s00702-010-0539-8

29. Schilder CM, Schagen SB. Effects of hormonal therapy on cognitive functioning in breast cancer patients: A review of the literature. Minerva Ginecol 2007;59:387401.

30. Dumitriu D, Rapp PR, McEwen BS, Morrison $\mathrm{JH}$. Estrogen and the aging brain: An elixir for the weary cortical network. Ann N Y Acad Sci. 2010; 1204: 104-12. Doi: 10.1111/j.1749-6632.2010.05529.x

31. Nelson CJ, Lee JS, Gamboa MC, Roth AJ. Cognitive effects of hormone therapy in 
men with prostate cancer. A review. Cancer 2008;113:1097-106. Doi:10.1002/ cncr.23658

33. Sundermann EE, Maki PM, Bishop JR. A review of estrogen receptor alpha gene (ESR1) polymorphisms, mood, and cognition. Menopause 2010;17:874-86. Doi:10.1097/gme.0b013e3181df4a19

33. Yaffe K, Edwards ER, Lui LY, Zmuda JM, Ferrell RE, Cauley JA Androgen receptor CAG repeat polymorphism is associated with cognitive function in older men. Biol Psychiatry. 2003;54:943-6. Doi:10.1016/ S0006-3223(03)00115-X

34. Ahles TA, Saykin AJ, McDonald BC, Li Y, Furstenberg CT, Hanscom BS, et al. Longitudinal assessment of cognitive changes associated with adjuvant treatment for breast cancer: impact of age and cognitive reserve. J Clin Oncol 2010;28:4434-40. Doi:10.1200/JCO.2009.27.0827

35. Shilling V, Jenkins V, Fallowfield L, Howell $\mathrm{T}$. The effects of hormone therapy on cognition in breast cancer. J Steroid Biochem Mol Biol. 2003;86:405-12. Doi:10.1016/j. jsbmb.2003.07.001

36. Jenkins VA, Ambroisine LM, Atkins L, Cuzick J, Howell A, Fallowfield LJ. Effects of anastrozole on cognitive performance in postmenopausal women: a randomised, double-blind chemoprevention trial (IBIS II). Lancet Oncol. 2008;9:953-61. Doi:10.1016/S1470-2045(08)70207-9

37. Schilder CM, Seynaeve C, Beex LV, Boogerd W, Linn SC, Gundy CM, et al. Effects of tamoxifen and examestane on cognitive function of postmenopausal patients with breast cancer: Results from neuropsychological side study of the Tamoxifen and Examestane Adjuvant Multinacional Trial. J Clin Oncol 2010;28:1294-300. Doi:10.1200/JCO.2008.21.3553

38. Alibhai SM, Breunis $H$, Timilshina $N$, Johnston C, Tomlinson G, Tannock I, et al. Impact of androgen-deprivation therapy on physical function and quality of life in men with nonmetastatic prostate cancer. J Clin
Oncol 2010;28:5038-45. Doi:10.1200/ JCO.2010.29.8091

39. Jacobsen PB, Garland LL, Booth-Jones M, Donovan KA, Thors $\mathrm{CL}$, Winters $\mathrm{E}$, et al. Relationship of haemoglobin levels to fatigue and cognitive functioning among cancer patients receiving chemotherapy. J Pain symptom Manage 2004;28:7-18. Doi:10.1016/j.jpainsymman.2003.11.002

40. López S, Cruzado JA, Feliu J. Rendimiento cognitivo, estado emocional y calidad de vida en pacientes con cáncer de colon previamente a recibir tratamiento de quimioterapia. Psicooncología 2009;6:121-37.

41. Mulhern RK, Fairclough D, Ochs J. A prospective comparison of neuropsychologic performance of children surviving leukemia who received 18-Gy, 24-Gy, or no cranial irradiation. J Clin Oncol. 1991;9:1348-56.

42. Silber JH, Radcliffe J, Peckham V, Perilongo G, Kishnani P, Fridman M, et al. Wholebrain irradiation and decline in intelligence: The influence of dose and age on IQ score. J Clin Oncol 1992;10:1390-96.

43. Dietrich J, Monje M, Wefel J, Meyers C. Clinical patterns and biological correlates of cognitive dysfunction associated with cancer therapy. Oncologist 2008;13:128595. Doi:10.1634/theoncologist.2008-0130

44. Welzel G, Fleckenstein K, Schaefer J, Hermann B, Kraus-Tiefenbacher U, Mai SK, et al. Memory function before and after whole brain radiotherapy in patients with and without brain metastases. Int J Radiat Oncol Biol Phys 2008;72:1311-18. Doi:10.1016/j.ijrobp.2008.03.009

45. Grossshans DR, Meyers CA, Allen PK, Davenport SD, Komaki R. Neurocognitive function in patients with small cell lung cancer. Effect of profilactic cranial irradiation. Cancer 2008;112:589-95. Doi:10.1002/ cncr.23222

46. Sun A, Bae K, Gore EM, Movsas B, Wong SJ, Meyers CA, et al. Phase III trial of prophylactic cranial irradiation compared with observation in patients with locally advanced non-small-cell lung cancer: Neu- 
rocognitive and quality-of-life analysis. J Clin Oncol 2011;29:279-86. Doi:10.1200/ JCO.2010.29.6053

47. Gehring K, Sitskoorn MM, Aaronson NK, Taphoorn MJB. Interventions for cognitive deficits in adults with brain tumours. Lancet Neurol 2008; 7: 548-60. Doi: $10.1177 / 0269215510395791$

48. Chang EL, Wefel JS, Hess KR, Allen PK, Lang FF, Kornguth DG, et al. Neurocognition in patients with brain metastases treated with radiosurgery or radiosurgery plus wholebrain irradiation: a randomised controlled trial. Lancet Oncol. 2009; 10: 1037-44. Doi:10.1016/S1470-2045(09)70263-3

49. Laack NN, Brown PD. Cognitive sequelae of brain radiation in adults. Semin Oncol 2004; 31: 702-13. Doi:10.1053/j.seminoncol.2004.07.013
50. Raber J. Unintended effects of cranial irradiation on cognitive function. Toxicol Pathol 2010; 38: 198-202. Doi:10.1177/0192623309352003

51. Monje ML, Mizumatsu S, Fike JR, Palmer TD. Irradiation induces neural precursorcell dysfunction. Nat Med 2002; 8: 955-62. Doi:10.1038/nm749

52. Monje ML, Toda H, Palmer TD. Inflamatory blockade restores adult hippocampal neurogenesis. Science 2003; 5651: 17605. Doi:10.1126/science.1088417

53. Monje ML, Vogel $\mathrm{H}$, Masek M, Ligon KL, Fisher PG, Palmer TD. Impaired human hippocampal neurogenesis after treatment for central nervous system malignancies. Ann Neurol 2007; 62: 515-20. Doi:10.1002/ ana.21214 
